\title{
Inflation from supersymmetry breaking
}

\section{Ignatios Antoniadis*}

Laboratoire de Physique Théorique et Hautes Énergies - LPTHE

Sorbonne Université, CNRS, 4 Place Jussieu, 75005 Paris, France

and

Albert Einstein Center, Institute for Theoretical Physics

University of Bern, Sidlerstrasse 5, 3012 Bern, Switzerland

E-mail: antoniadiseitp.unibe.ch

\begin{abstract}
I discuss a general class of models where the inflation is driven by supersymmetry breaking with the superpartner of the goldstino (sgoldstino) playing the role of the inflaton. Imposing an Rsymmetry allows to satisfy easily the slow-roll conditions, avoiding the so-called $\eta$-problem, and leads to two different classes of small field inflation models; they are characterised by an inflationary plateau around the maximum of the scalar potential, where R-symmetry is either restored or spontaneously broken, with the inflaton rolling down to a minimum describing the present phase of our Universe. Inflation can be driven by either an F- or a D-term, while the minimum has a positive tuneable vacuum energy. The models agree with cosmological observations and in the simplest case predict a tensor-to-scalar ratio of primordial perturbations $10^{-9} \lesssim r \lesssim 10^{-4}$ and an inflation scale $10^{10} \mathrm{GeV} \lesssim H_{*} \lesssim 10^{12} \mathrm{GeV}$.
\end{abstract}

Corfu Summer Institute 2018 "School and Workshops on Elementary Particle Physics and Gravity" (CORFU2018)

31 August - 28 September, 2018

Corfu, Greece

${ }^{*}$ Speaker. 


\section{Introduction}

If String Theory is a fundamental theory of Nature and not just a tool for studying systems with strongly coupled dynamics, it should be able to describe at the same time particle physics and cosmology, which are phenomena that involve very different scales from the microscopic fourdimensional (4d) quantum gravity length of $10^{-33} \mathrm{~cm}$ to large macroscopic distances of the size of the observable Universe $\sim 10^{28} \mathrm{~cm}$ spanned a region of about 60 orders of magnitude. In particular, besides the $4 \mathrm{~d}$ Planck mass, there are three very different scales with very different physics corresponding to the electroweak, dark energy and inflation. These scales might be related via the scale of the underlying fundamental theory, such as string theory, or they might be independent in the sense that their origin could be based on different and independent dynamics. An example of the former constraint and more predictive possibility is provided by $\mathrm{TeV}$ strings with a fundamental scale at low energies due for instance to large extra dimensions transverse to a four-dimensional braneworld forming our Universe [1]. In this case, the $4 \mathrm{~d}$ Planck mass is emergent from the fundamental string scale and inflation should also happen around the same scale [2].

Here, we will adopt a more conservative approach, trying to relate the scales of supersymmetry breaking and inflation, assuming that supersymmetry breaking is realised in a metastable de Sitter vacuum with an infinitesimally small (tuneable) cosmological constant independent of the breaking scale that may be in the $\mathrm{TeV}$ region or higher.

In a recent work [3], we studied a simple $N=1$ supergravity model having this property and motivated by string theory. Besides the gravity multiplet, the minimal field content consists of a chiral multiplet with a shift symmetry promoted to a gauged R-symmetry using a vector multiplet. In the string theory context, the chiral multiplet can be identified with the string dilaton (or an appropriate compactification modulus) and the shift symmetry associated to the gauge invariance of a two-index antisymmetric tensor that can be dualized to a (pseudo)scalar. The shift symmetry fixes the form of the superpotential and the gauging allows for the presence of a Fayet-Iliopoulos (FI) term [4], leading to a supergravity action with two independent parameters that can be tuned so that the scalar potential possesses a metastable de Sitter minimum with a tiny vacuum energy (essentially the relative strength between the F- and D-term contributions). A third parameter fixes the Vacuum Expectation Value (VEV) of the string dilaton at the desired (phenomenologically) weak coupling regime. An important consistency constraint of the model is anomaly cancellation which has been studied in [5] and implies the existence of additional charged fields under the gauged R-symmetry.

In a subsequent work [6], we analysed a small variation of this model which is manifestly anomaly free without additional charged fields and allows to couple in a straight forward way a visible sector containing the minimal supersymmetric extension of the Standard Model (MSSM) and studied the mediation of supersymmetry breaking and its phenomenological consequences. It turns out that an additional 'hidden sector' field $z$ is needed to be added for the matter soft scalar masses to be non-tachyonic; although this field participates in the supersymmetry breaking and is similar to the so-called Polonyi field, it does not modify the main properties of the metastable de Sitter (dS) vacuum. All soft scalar masses, as well as trilinear A-terms, are generated at the tree level and are universal under the assumption that matter kinetic terms are independent of the 'Polonyi' field, since matter fields are neutral under the shift symmetry and supersymmetry break- 
ing is driven by a combination of the $U(1) \mathrm{D}$-term and the dilaton and $z$-field F-term. Alternatively, a way to avoid the tachyonic scalar masses without adding the extra field $z$ is to modify the matter kinetic terms by a dilaton dependent factor.

A main difference of the second analysis from the first work is that we use a field representation in which the gauged shift symmetry corresponds to an ordinary $U(1)$ and not an R-symmetry. The two representations differ by a Kähler transformation that leaves the classical supergravity action invariant. However, at the quantum level, there is a Green-Schwarz term generated that amounts an extra dilaton dependent contribution to the gauge kinetic terms needed to cancel the anomalies of the R-symmetry. This creates an apparent puzzle with the gaugino masses that vanish in the first representation but not in the latter. The resolution to the puzzle is based on the so called anomaly mediation contributions $[7,8]$ that explain precisely the above apparent discrepancy. It turns out that gaugino masses are generated at the quantum level and are thus suppressed compared to the scalar masses (and A-terms).

This model has the necessary ingredients to be obtained as a remnant of moduli stabilisation within the framework of internal magnetic fluxes in type I string theory, turned on along the compact directions for several abelian factors of the gauge group. All geometric moduli can in principle be fixed in a supersymmetric way, while the shift symmetry is associated to the $4 \mathrm{~d}$ axion and its gauging is a consequence of anomaly cancellation $[9,10]$.

We then made an attempt to connect the scale of inflation with the electroweak and supersymmetry breaking scales within the same effective field theory, that at the same time allows the existence of an infinitesimally small (tuneable) positive cosmological constant describing the present dark energy of the universe. We thus addressed the question whether the same scalar potential can provide inflation with the dilaton playing also the role of the inflaton at an earlier stage of the universe evolution [11]. We showed that this is possible if one modifies the Kähler potential by a correction that plays no role around the minimum, but creates an appropriate plateau around the maximum. In general, the Kähler potential receives perturbative and non-perturbative corrections that vanish in the weak coupling limit. After analysing all such corrections, we find that only those that have the form of (Neveu-Schwarz) NS5-brane instantons can lead to an inflationary period compatible with cosmological observations. The scale of inflation turns out then to be of the order of low energy supersymmetry breaking, in the $\mathrm{TeV}$ region. On the other hand, the predicted tensor-to-scalar ratio is too small to be observed.

Inflationary models [12] in supergravity ${ }^{1}$ suffer in general from several problems, such as finetuning to satisfy the slow-roll conditions, large field initial conditions that break the validity of the effective field theory, and stabilisation of the (pseudo) scalar companion of the inflaton arising from the fact that bosonic components of superfields are always even. The simplest argument to see the fine tuning of the potential is that a canonically normalised kinetic term of a complex scalar field $X$ corresponds to a quadratic Kähler potential $K=X \bar{X}$ that brings one unit contribution to the slowroll parameter $\eta=V^{\prime \prime} / V$, arising from the $e^{K}$ proportionality factor in the expression of the scalar potential $V$. This problem can be avoided in models with no-scale structure where cancellations arise naturally due to non-canonical kinetic terms leading to potentials with flat directions (at the classical level). However, such models require often trans-Planckian initial conditions that inval-

\footnotetext{
${ }^{1}$ For reviews on supersymmetric models of inflation, see for example [13].
} 
idate the effective supergravity description during inflation. A concrete example where all these problems appear is the Starobinsky model of inflation [14], despite its phenomenological success.

All three problems above are solved when the inflaton is identified with the scalar component of the goldstino superfield ${ }^{2}$, in the presence of a gauged R-symmetry [16]. Indeed, the superpotential is in that case linear and the big contribution to $\eta$ described above cancels exactly. Since inflation arises at a plateau around the maximum of the scalar potential (hill-top) no large field initial conditions are needed, while the pseudo-scalar companion of the inflaton is absorbed into the R-gauge field that becomes massive, leading the inflaton as a single scalar field present in the low-energy spectrum. This model provides therefore a minimal realisation of natural small-field inflation in supergravity, compatible with present observations, as we show below. Moreover, it allows the presence of a realistic minimum describing our present Universe with an infinitesimal positive vacuum energy arising due to a cancellation between an F- and D-term contributions to the scalar potential, without affecting the properties of the inflationary plateau, along the lines of Refs. [3, 11, 17].

In the above models the D-term has a constant FI contribution but plays no role during inflation and can be neglected, while the pseudoscalar partner of the inflaton is absorbed by the $U(1)_{R}$ gauge field that becomes massive away from the origin. Recently, a new FI term was proposed [19] that has three important properties: (1) it is manifestly gauge invariant already at the Lagrangian level; (2) it is associated to a $U(1)$ that should not gauge an R-symmetry and (3) supersymmetry is broken by (at least) a D-auxiliary expectation value and the extra bosonic part of the action is reduced in the unitary gauge to a constant FI contribution leading to a positive shift of the scalar potential, in the absence of matter fields. In the presence of matter fields, the FI contribution to the D-term acquires a special field dependence $e^{2 K / 3}$ that violates invariance under Kähler transformations.

In a recent work [18], we studied the properties of the new FI term and explored its consequences to the class of inflation models we introduced in [16]. ${ }^{3}$ We first showed that matter fields charged under the $U(1)$ gauge symmetry can consistently be added in the presence of the new FI term, as well as a non-trivial gauge kinetic function. We then observed that the new FI term is not invariant under Kähler transformations. On the other hand, a gauged R-symmetry in ordinary Kähler invariant supergravity can always be reduced to an ordinary (non-R) $U(1)$ by a Kähler transformation. By then going to such a frame, we find that the two FI contributions to the $U(1) \mathrm{D}$-term can coexist, leading to a novel contribution to the scalar potential.

The resulting D-term scalar potential provides an alternative realisation of inflation from supersymmetry breaking, driven by a D- instead of an F-term. The inflaton is still a superpartner of the goldstino which is now a gaugino within a massive vector multiplet, where again the pseudoscalar partner is absorbed by the gauge field away from the origin. For a particular choice of the inflaton charge, the scalar potential has a maximum at the origin where inflation occurs and a supersymmetric minimum at zero energy, in the limit of negligible F-term contribution (such as in the absence of superpotential). The slow roll conditions are automatically satisfied near the point where the new FI term cancels the charge of the inflaton, leading to higher than quadratic contributions due to its non trivial field dependence.

\footnotetext{
${ }^{2}$ See [15] for earlier work relating supersymmetry and inflation.

${ }^{3}$ This new FI term was also studied in [20] to remove an instability from inflation in Polonyi-Starobinsky supergravity.
} 
The Kähler potential can be canonical, modulo the Kähler transformation that takes it to the non R-symmetry frame. In the presence of a small superpotential, the inflation is practically unchanged and driven by the D-term, as before. However, the maximum is now slightly shifted away from the origin and the minimum has a small non-vanishing positive vacuum energy, where supersymmetry is broken by both F- and D-auxiliary expectation values of similar magnitude. The model predicts in general small primordial gravitational waves with a tensor-to-scaler ration $r$ well below the observability limit. However, when higher order terms are included in the Kähler potential, one finds that $r$ can increase to large values $r \simeq 0.015$.

On general grounds, there are two classes of such models depending on whether the maximum corresponds to a point of unbroken (case 1) or broken (case 2) R-symmetry. The latter corresponds actually to a generalisation of the model we discussed above [11], inspired by string theory [3]. It has the same field content but in a different field basis with a chiral multiplet $S \propto \ln X$ playing the role of the string dilaton. Thus, $S$ has a shift symmetry which is actually an R-symmetry gauged by a vector multiplet and the superpotential is a single exponential. The scalar potential has a minimum with a tuneable vacuum energy and a maximum that can produce inflation when appropriate corrections are included in the Kähler potential. In these coordinates R-symmetry is restored at infinity, corresponding to the weak coupling limit. Small field inflation is again guaranteed consistently with the validity of the effective field theory.

In the following, we will present the main features of models of case 1, where inflation occurs near the maximum of the scalar potential where R-symmetry is restored and supersymmetry breaking is driven predominantly either by an F-term or by a D-term.

\section{Conventions}

Throughout this paper we use the conventions of [21]. A supergravity theory is specified (up to Chern-Simons terms) by a Kähler potential $\mathscr{K}$, a superpotential $W$, and the gauge kinetic functions $f_{A B}(z)$. The chiral multiplets $z^{\alpha}, \chi^{\alpha}$ are enumerated by the index $\alpha$ and the indices $A, B$ indicate the different gauge groups. Classically, a supergravity theory is invariant under Kähler tranformations, viz.

$$
\begin{aligned}
\mathscr{K}(z, \bar{z}) & \longrightarrow \mathscr{K}(z, \bar{z})+J(z)+\bar{J}(\bar{z}), \\
W(z) & \longrightarrow e^{-\kappa^{2} J(z)} W(z),
\end{aligned}
$$

where $\kappa$ is the inverse of the reduced Planck mass, $M_{\mathrm{Pl}}=\kappa^{-1}=2.4 \times 10^{15} \mathrm{TeV}$. The gauge transformations of chiral multiplet scalars are given by holomorphic Killing vectors, i.e. $\delta z^{\alpha}=\theta^{A} k_{A}^{\alpha}(z)$, where $\theta^{A}$ is the gauge parameter of the gauge group $A$. The Kähler potential and superpotential need not be invariant under this gauge transformation, but can change by a Kähler transformation

$$
\delta \mathscr{K}=\theta^{A}\left[r_{A}(z)+\bar{r}_{A}(\bar{z})\right]
$$

provided that the gauge transformation of the superpotential satisfies $\delta W=-\theta^{A} \kappa^{2} r_{A}(z) W$. One then has from $\delta W=W_{\alpha} \delta z^{\alpha}$

$$
W_{\alpha} k_{A}^{\alpha}=-\kappa^{2} r_{A} W,
$$


where $W_{\alpha}=\partial_{\alpha} W$ and $\alpha$ labels the chiral multiplets. The supergravity theory can then be described by a gauge invariant function

$$
\mathscr{G}=\kappa^{2} \mathscr{K}+\log \left(\kappa^{6} W \bar{W}\right) .
$$

The scalar potential is given by

$$
\begin{aligned}
V & =V_{F}+V_{D} \\
V_{F} & =e^{\kappa^{2} \mathscr{K}}\left(-3 \kappa^{2} W \bar{W}+\nabla_{\alpha} W g^{\alpha \bar{\beta}} \bar{\nabla}_{\bar{\beta}} \bar{W}\right) \\
V_{D} & =\frac{1}{2}(\operatorname{Re} f)^{-1 A B} \mathscr{P}_{A} \mathscr{P}_{B},
\end{aligned}
$$

where W appears with its Kähler covariant derivative

$$
\nabla_{\alpha} W=\partial_{\alpha} W(z)+\kappa^{2}\left(\partial_{\alpha} \mathscr{K}\right) W(z) .
$$

The moment maps $\mathscr{P}_{A}$ are given by

$$
\mathscr{P}_{A}=i\left(k_{A}^{\alpha} \partial_{\alpha} \mathscr{K}-r_{A}\right) .
$$

In this paper we will be concerned with theories having a gauged R-symmetry, for which $r_{A}(z)$ is given by an imaginary constant $r_{A}(z)=i \kappa^{-2} \xi$. In this case, $\kappa^{-2} \xi$ is a Fayet-Iliopoulos [4] constant parameter.

\section{Symmetric versus non-symmetric point}

Here, we present a class of inflation models in supergravity theories containing a single chiral multiplet transforming under a gauged R-symmetry with a corresponding abelian vector multiplet [16]. We assume that the chiral multiplet $\mathscr{X}$ (with scalar component $X$ ) transforms as:

$$
X \longrightarrow X e^{-i q \omega} \text {. }
$$

where $q$ is its charge, and $\omega$ is the gauge parameter.

The Kähler potential is therefore a function of $X \bar{X}$, while the superpotential is constrained to be of the form $X^{b}$ :

$$
\begin{aligned}
\mathscr{K} & =\mathscr{K}(X \bar{X}), \\
W & =\kappa^{-3} f X^{b},
\end{aligned}
$$

where $X$ is a dimensionless field. For $b \neq 0$, the gauge symmetry eq. (3.1) becomes a gauged Rsymmetry. The gauge kinetic function can have a constant contribution as well as a contribution proportional to $\ln X$

$$
f(X)=\gamma+\beta \ln X .
$$

The latter contribution proportional to $\beta$ is not gauge invariant and can be used as a Green-Schwarz counter term to cancel possible anomalies. One can show however that the constant $\beta$ is fixed to be very small by anomaly cancellation conditions and does not change our results [16]. We will therefore omit this term in our analysis below. 
We are interested in the general properties of supergravity theories of inflation that are of the above form. Before performing our analysis, a distinction should be made concerning the initial point where slow-roll inflation starts. The inflaton field (which will turn out to be $\rho$, where $X=\rho e^{i \theta}$ ) can either have its initial value close to the symmetric point where $X=0$, or at a generic point $X \neq 0$. The minimum of the potential, however, is always at a nonzero point $X \neq 0$. This is because at $X=0$ the negative contribution to the scalar potential vanishes and no cancellation between F-term and D-term is possible. The supersymmetry breaking scale is therefore related to the cosmological constant as $\kappa^{-2} m_{3 / 2}^{2} \approx \Lambda$. One could in principle assume that the value of the potential at its minimum is of the order of the supersymmetry breaking scale. However, in this case additional corrections are needed to bring down the minimum of the potential to the present value of the cosmological constant, and we therefore do not discuss this possibility.

In the first case, inflation starts near $X=0$, and the inflaton field will roll towards a minimum of the potential at $X \neq 0$. On the other hand, in the second case inflation will start at a generic point $X \neq 0$. It is then convenient to work with another chiral superfield $S$, which is invariant under a shift symmetry

$$
S \longrightarrow S-i c \alpha
$$

by performing a field redefinition

$$
X=e^{S}
$$

In this case the most general Kähler potential and superpotential are of the form

$$
\begin{aligned}
\mathscr{K} & =\mathscr{K}(S+\bar{S}), \\
W & =\kappa^{-3} a e^{b S} .
\end{aligned}
$$

Note that this field redefinition is not valid at the symmetric point $X=0$ for the first case.

\section{Case 1: Inflation near the symmetric point}

\subsection{Slow roll parameters}

In this section we derive the conditions that lead to slow-roll inflation scenarios, where the start of inflation is near a local maximum of the potential at $X=0$. Since the superpotential has charge 2 under R-symmetry, one has $\langle W\rangle=0$ as long as R-symmetry is preserved. Therefore, $\langle W\rangle$ can be regarded as the order parameter of R-symmetry breaking. On the other hand, the minimum of the potential requires $\langle W\rangle \neq 0$ and broken R-symmetry. It is therefore attractive to assume that at earlier times R-symmetry was a good symmetry, switching off dangerous corrections to the potential. As similar approach was followed in [22], where a discrete R-symmetry is assumed. Instead, we assume a gauged R-symmetry which is spontaneously broken at the minimum of the potential.

While the superpotential is uniquely fixed in eq. (3.2), the Kähler potential is only fixed to be of the form $\mathscr{K}(X \bar{X})$. We expand the Kähler potential as follows

$$
\begin{aligned}
\mathscr{K}(X, \bar{X}) & =\kappa^{-2} X \bar{X}+\kappa^{-2} A(X \bar{X})^{2}, \\
W(X) & =\kappa^{-3} f X^{b}, \\
f(X) & =1
\end{aligned}
$$


where $A$ and $f$ are constants. The gauge kinetic function is taken to be constant since it was shown that the coefficient $\beta$ in front of the logarithmic term in eq. (3.3) is fixed to be very small by anomaly cancellation conditions [16]. As far as the scalar potential is concerned, the coefficient $\gamma$ can be absorbed in other parameters of the theory. We therefore take $\gamma=1$.

The scalar potential is given by

$$
\mathscr{V}=\mathscr{V}_{F}+\mathscr{V}_{D}
$$

where

$$
\mathscr{V}_{F}=\kappa^{-4} f^{2}(X \bar{X})^{b-1} e^{X \bar{X}(1+A X \bar{X})}\left[-3 X \bar{X}+\frac{(b+X \bar{X}(1+2 A X \bar{X}))^{2}}{1+4 A X \bar{X}}\right]
$$

and

$$
\mathscr{V}_{D}=\kappa^{-4} \frac{q^{2}}{2}[b+X \bar{X}(1+2 A X \bar{X})]^{2} .
$$

The superpotential is not gauge invariant under the $U(1)$ gauge symmetry. Instead it transforms as

$$
W \rightarrow W e^{-i q b w}
$$

Therefore, the $U(1)$ is a gauged R-symmetry which we will further denote as $U(1)_{R}$. From $W_{X} k_{R}^{X}=$ $-r_{R} \kappa^{2} W$, where $k_{R}^{X}=-i q X$ is the Killing vector for the field $X$ under the R-symmetry, $r_{R}=$ $i \kappa^{-2} \xi_{R}$ with $\kappa^{-2} \xi_{R}$ the Fayet-Iliopoulos contribution to the scalar potential, and $W_{X}$ is short-hand for $\partial W / \partial X$, we find

$$
r_{R}=i \kappa^{-2} q b
$$

A consequence of the gauged R-symmetry is that the superpotential coupling $b$ enters the $\mathrm{D}$-term contribution of the scalar potential as a constant Fayet-Iliopoulos contribution. ${ }^{4}$

Note that the scalar potential is only a function of the modulus of $X$ and that the potential contains a Fayet-Iliopoulos contribution for $b \neq 0$. Moreover, its phase will be 'eaten' by the $U(1)$ gauge boson upon a field redefinition of the gauge potential similarly to the standard Higgs mechanism. After performing a change of field variables

$$
X=\rho e^{i \theta}, \quad \bar{X}=\rho e^{-i \theta}, \quad(\rho \geq 0)
$$

the scalar potential is a function of $\rho$,

$$
\kappa^{4} \mathscr{V}=f^{2} \rho^{2(b-1)} e^{\rho^{2}+A \rho^{4}}\left(-3 \rho^{2}+\frac{\left(b+\rho^{2}+2 A \rho^{4}\right)^{2}}{1+4 A \rho^{2}}\right)+\frac{q^{2}}{2}\left(b+\rho^{2}+2 A \rho^{4}\right)^{2} .
$$

Since we assume that inflation starts near $\rho=0$, we require that the potential eq. (4.8) has a local maximum at this point. It turns out that the potential only allows for a local maximum at $\rho=0$

\footnotetext{
${ }^{4}$ For other studies of inflation involving Fayet-Iliopoulos terms see for example [24], or [25] for more recent work. Moreover, our motivations have some overlap with [22], where inflation is also assumed to start near an R-symmetric point at $X=0$. However, this work uses a discrete R-symmetry which does not lead to Fayet-Iliopoulos terms.
} 
when $b=1$. For $b<1$ the potential diverges when $\rho$ goes to zero. For $1<b<1.5$ the first derivative of the potential diverges, while for $b=1.5$, one has $V^{\prime}(0)=\frac{9}{4} f^{2}+\frac{3}{2} q^{2}>0$, and for $b>1.5$, on has $V^{\prime \prime}(0)>0$. We thus take $b=1$ and the scalar potential reduces to

$$
\kappa^{4} \mathscr{V}=f^{2} e^{\rho^{2}+A \rho^{4}}\left(-3 \rho^{2}+\frac{\left(1+\rho^{2}+2 A \rho^{4}\right)^{2}}{1+4 A \rho^{2}}\right)+\frac{q^{2}}{2}\left(1+\rho^{2}+2 A \rho^{4}\right)^{2} .
$$

A plot of the potential for $A=1 / 2, q=1$ and $f$ tuned so that the minimum has zero energy is given in Figure 1.

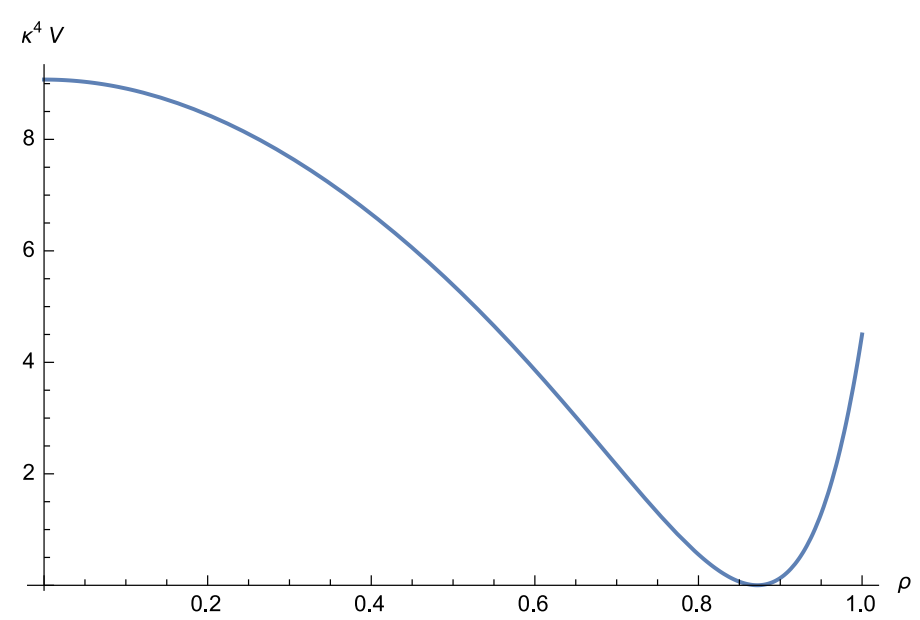

Figure 1:

Note that in this case the the superpotential is linear $W=f X$, describing the sgoldstino (up to an additional low-energy constraint) [26]. Indeed, modulo a D-term contribution, the inflaton in this model is the superpartner of the goldstino. In fact, for $q=0$ the inflaton reduces to the partner of the goldstino as in Minimal Inflation models [27]. The important difference however is that this is a microscopic realisation of the identification of the inflaton with the sgoldstino, and that the so-called $\eta$-problem is avoided (see discussion below).

The kinetic terms for the scalars can be written as ${ }^{5}$

$$
\begin{aligned}
\mathscr{L}_{\text {kin }} & =-g_{X \bar{X}} \hat{\partial}_{\mu} X \hat{\partial}^{\mu} X \\
& =-g_{X \bar{X}}\left[\partial_{\mu} \rho \partial^{\mu} \rho+\rho^{2}\left(\partial_{\mu} \theta+q A_{\mu}\right)\left(\partial^{\mu} \theta+q A^{\mu}\right)\right] .
\end{aligned}
$$

It was already anticipated above that the phase $\theta$ plays the role of the longitudinal component of the gauge field $A_{\mu}$, which acquires a mass by a Brout-Englert-Higgs mechanism.

We now interpret the field $\rho$ as the inflaton. It is important to emphasise that, in contrast with usual supersymmetric theories of inflation where one necessarily has two scalar degrees of freedom resulting in multifield inflation [28], our class of models contains only one scalar field $\rho$ as the inflaton. In order to calculate the slow-roll parameters, one needs to work with the canonically

\footnotetext{
${ }^{5}$ The covariant derivative is defined as $\hat{\partial}_{\mu} X=\partial_{\mu} X-A_{\mu} k_{R}^{X}$, where $k_{R}^{X}=-i q X$ is the Killing vector for the $\mathrm{U}(1)$ transformation eq. (3.1).
} 
normalised field $\chi$ satisfying

$$
\frac{d \chi}{d \rho}=\sqrt{2 g_{X \bar{X}}}
$$

The slow-roll parameters are given in terms of the canonical field $\chi$ by

$$
\varepsilon=\frac{1}{2 \kappa^{2}}\left(\frac{d V / d \chi}{V}\right)^{2}, \quad \eta=\frac{1}{\kappa^{2}} \frac{d^{2} V / d \chi^{2}}{V} .
$$

Since we assume inflation to start near $\rho=0$, we expand

$$
\begin{aligned}
& \varepsilon=4\left(\frac{-4 A+x^{2}}{2+x^{2}}\right)^{2} \rho^{2}+\mathscr{O}\left(\rho^{4}\right), \\
& \eta=2\left(\frac{-4 A+x^{2}}{2+x^{2}}\right)+\mathscr{O}\left(\rho^{2}\right),
\end{aligned}
$$

where we defined $q=f x$. Notice that for $\rho \ll 1$ the $\varepsilon$ parameter is very small, while the $\eta$ parameter can be made small by carefully tuning the parameter $A$. Any higher order corrections to the Kähler potential do not contribute to the leading contributions in the expansion near $\rho=0$ for $\eta$ and $\varepsilon$. Such corrections can therefore be used to alter the potential near its minimum, at some point $X \neq 0$ without influencing the slow-roll parameters.

\section{A comment on the $\eta$-problem in Supergravity}

A few words are now in order concerning the $\eta$-problem [29]. The $\eta$ problem in $\mathscr{N}=1$ supergravity is often stated as follows (see for example [30]): If, for instance, a theory with a single chiral multiplet with scalar component $\varphi$ is taken, then the Kähler potential can be expanded around a reference location $\varphi=0$ as $\mathscr{K}=\mathscr{K}(0)+\mathscr{K}_{\varphi \bar{\varphi}}(0) \varphi \bar{\varphi}+\ldots$. The Lagrangian becomes

$$
\mathscr{L}=-\partial_{\mu} \phi \partial^{\mu} \bar{\phi}-\mathscr{V}(0)\left(1+\kappa^{2} \phi \bar{\phi}+\cdots\right),
$$

where $\phi$ is the canonically normalised field $\phi \bar{\phi}=\mathscr{K}_{\varphi \bar{\varphi}}(0) \varphi \bar{\varphi}$, and the ellipses stand for extra terms in the expansion coming from $\mathscr{K}$ and $W$. Following this argument, the mass $m_{\phi}$ turns out to be proportional to the Hubble scale

$$
m_{\phi}^{2}=\kappa^{2} \mathscr{V}(0)+\cdots=3 H^{2}+\cdots
$$

and therefore

$$
\eta=\frac{m_{\phi}^{2}}{3 H^{2}}=1+\ldots
$$

Or otherwise stated, this leading contribution of order 1 to the $\eta$-parameter has its origin from the fact that the F-term contribution to the scalar potential contains an exponential factor $e^{\mathscr{K}}$ : $\mathscr{V}=e^{X \bar{X}+\ldots}[\ldots]$ resulting in its second derivative $\mathscr{V}_{X \bar{X}}=V[1+\ldots]$.

However, in our model the factor ' 1 ' drops out for the particular choice $b=1$ in the superpotential ${ }^{6}$, resulting in an inflaton mass $m_{\rho}^{2}$ which is determined by the next term $A(X \bar{X})^{2}$ in the

\footnotetext{
${ }^{6}$ Note that in hybrid inflation models the $\eta$-problem is also evaded by a somewhat similar way, but these models generally include several scalar fields (and superfields) besides the inflaton (see e.g. [31]).
} 
expansion of the Kähler potential,

$$
\begin{aligned}
& m_{\chi}^{2}=\left(-4 A+x^{2}\right) \kappa^{-2} f^{2}+\mathscr{O}\left(\rho^{2}\right), \\
& H^{2}=\frac{\kappa^{-2} f^{2}}{6}\left(2+x^{2}\right)+\mathscr{O}\left(\rho^{2}\right) .
\end{aligned}
$$

As a result, there are two ways to evade the $\eta$-problem:

- First, one can obtain a small $\eta$ by having a small $q \ll f$, while $A$ should be of order $\mathscr{O}\left(10^{-1}\right)$. In this case, the rôle of the gauge symmetry is merely to constrain the form of the Kähler potential and the superpotential, and to provide a Higgs mechanism that eliminates the extra scalar (phase) degree of freedom.

- Alternatively there could be a cancellation between $q^{2}$ and $4 A f^{2}$.

Since $A$ is the second term in the expansion of the Kähler potential eq. (4.1), it is natural to be of order $\mathscr{O}\left(10^{-1}\right)$ and therefore providing a solution to the $\eta$-problem.

Note that the mass of the inflaton given in eqs. (4.17) is only valid during inflation at small $\rho$. The mass of the inflaton at its VEV will be affected by additional corrections that are needed to obtain in particular a vanishing value for the scalar potential at its minimum [16].

\section{The upper bound on the tensor-to-scalar ratio}

Before moving on to the next section, let us focus on the approximation at $\rho \ll 1$ where the perturbative expansion of the slow-roll parameters in eqs. (4.13) is valid, and assume that the horizon exit occurs at the field value $\rho_{*}$ very close to the maximum $\rho=0$. In this approximation, eqs. (4.13) become

$$
\varepsilon(\rho) \approx \varepsilon^{\text {pert }}(\rho)=\left|\eta_{*}\right|^{2} \rho^{2}, \quad \eta(\rho) \approx \eta_{*},
$$

where the asterisk refers to the value of parameters evaluated at the horizon exit.

To discuss the upper bound on the tensor-to-scalar ratio, it is convenient to divide the region $\left[\rho=0, \rho_{\text {end }}\right]$ into two regions: one is $\left[0, \rho_{\mathrm{p}}\right]$, where the approximation 4.18 is valid, and the other is the rest $\left[\rho_{\mathrm{p}}, \rho_{\mathrm{end}}\right]$. Here $\rho_{\mathrm{end}}$ means the inflation end. Note that $\rho_{\mathrm{p}}<\rho_{\text {end }}$ because the approximation 4.18 breaks down before the end of inflation where $\varepsilon\left(\rho_{\text {end }}\right)=1$ or $\left|\eta\left(\rho_{\text {end }}\right)\right|=1$. In terms of this division, the number of e-folds from the horizon exit to the end of inflation can be approximated by

$$
N_{\mathrm{CMB}} \simeq N^{\mathrm{pert}}\left(\rho_{*}, \rho_{\mathrm{p}}\right)+\kappa \int_{\chi_{\mathrm{p}}}^{\chi_{\text {end }}} \frac{d \chi}{\sqrt{2 \varepsilon(\chi)}},
$$

where we introduced

$$
N^{\text {pert }}\left(\rho_{1}, \rho_{2}\right)=\kappa \int_{\chi_{1}}^{\chi_{2}} \frac{d \chi}{\sqrt{2 \varepsilon^{\text {pert }}(\chi)}}=\frac{1}{\left|\eta_{*}\right|} \ln \left(\frac{\rho_{2}}{\rho_{1}}\right) .
$$

Here $\chi$ is the canonically normalised field defined by eq. (4.11). Let us next focus on the region $\left[\rho_{\mathrm{p}}, \rho_{\mathrm{end}}\right]$. It is natural to expect the following inequality

$$
\kappa \int_{\chi_{\mathrm{p}}}^{\chi_{\text {end }}} \frac{d \chi}{\sqrt{2 \varepsilon(\chi)}} \lesssim \kappa \int_{\chi_{\mathrm{p}}}^{\chi_{\text {end }}} \frac{d \chi}{\sqrt{2 \varepsilon^{\text {pert }}(\chi)}} .
$$


This is based on the following observation. The right hand side describes a hypothetical situation, as if the slow-roll condition were valid throughout the inflation until its end. But since in the actual inflation the slow-roll condition breaks down in the region $\left[\rho_{\mathrm{p}}, \rho_{\mathrm{end}}\right]$, the actual number of e-folds in this region will be smaller than that in the hypothetical situation. Adding $N^{\text {pert }}\left(\rho_{*}, \rho_{\mathrm{p}}\right)$ to the both hand sides of 4.21 and using 4.19 , we find

$$
N_{\mathrm{CMB}} \lesssim \frac{1}{\left|\eta_{*}\right|} \ln \left(\frac{\rho_{\mathrm{end}}}{\rho_{*}}\right) .
$$

Using 4.18 and the definition of the tensor-to-scalar ratio $r=16 \varepsilon_{*}$, we obtain the upper bound:

$$
r \lesssim 16\left(\left|\eta_{*}\right| \rho_{\mathrm{end}} e^{-\left|\eta_{*}\right| N_{\mathrm{CMB}}}\right)^{2}
$$

To satisfy CMB data, let us choose $\eta=-0.02$ and $N_{\mathrm{CMB}} \approx 50$. Assuming $\rho_{\text {end }} \lesssim 1 / 2$, we obtain the upper bound $r \lesssim 10^{-4}$. Note that this is a little bit lower than the Lyth bound [32] for small field inflation, $r \lesssim 10^{-3}$. From the upper bound on $r$, we can also find the upper bound on the Hubble parameter as follows. In general, the power spectrum amplitude $A_{s}$ is related to the Hubble parameter at horizon exit $H_{*}$ by

$$
A_{s}=\frac{2 \kappa^{2} H_{*}^{2}}{\pi^{2} r}
$$

Combining this with the upper bound $r \lesssim 10^{-4}$ and the value $A_{s}=2.2 \times 10^{-9}$ by CMB data, we find the upper bound on the Hubble parameter $H_{*} \lesssim 10^{9} \mathrm{TeV}$.

In Ref. [16], we will also find the lower bound $r \gtrsim 10^{-9}$ (equivalently $H_{*} \gtrsim 10^{7} \mathrm{TeV}$ ), based on an model-independent argument. This bound can be lowered at the cost of naturalness between parameters in the potential.

\section{On the new FI term}

\subsection{Review}

In [19], the authors propose a new contribution to the supergravity Lagrangian of the form ${ }^{7}$

$$
\mathscr{L}_{\mathrm{FI}}=\xi_{2}\left[S_{0} \bar{S}_{0} \frac{w^{2} \bar{w}^{2}}{\bar{T}\left(w^{2}\right) T\left(\bar{w}^{2}\right)}(V)_{D}\right]_{D}
$$

The chiral compensator field $S_{0}$, with Weyl and chiral weights (Weyl, Chiral) $=(1,1)$, has components $S_{0}=\left(s_{0}, P_{L} \Omega_{0}, F_{0}\right)$. The vector multiplet has vanishing Weyl and chiral weights, and its components are given by $V=\left(v, \zeta, \mathscr{H}, v_{\mu}, \lambda, D\right)$. In the Wess-Zumino gauge, the first components are put to zero $v=\zeta=\mathscr{H}=0$. The multiplet $w^{2}$ is of weights $(1,1)$, and given by

$$
w^{2}=\frac{\bar{\lambda} P_{L} \lambda}{S_{0}^{2}}, \quad \bar{w}^{2}=\frac{\lambda P_{R} \bar{\lambda}}{\bar{S}_{0}^{2}} .
$$

\footnotetext{
${ }^{7}$ A similar, but not identical term was studied in [33].
} 
The components of $\bar{\lambda} P_{L} \lambda$ are given by

$$
\bar{\lambda} P_{L} \lambda=\left(\bar{\lambda} P_{L} \lambda ; \sqrt{2} P_{L}\left(-\frac{1}{2} \gamma \cdot \hat{F}+i D\right) \lambda ; 2 \bar{\lambda} P_{L} \not \lambda+\hat{F}^{-} \cdot \hat{F}^{-}-D^{2}\right) .
$$

The kinetic terms for the gauge multiplet are given by

$$
\mathscr{L}_{\text {kin }}=-\frac{1}{4}\left[\bar{\lambda} P_{L} \lambda\right]_{F}+\text { h.c. } .
$$

The operator $T(\bar{T})$ is defined in [34, 35], and leads to a chiral (antichiral) multiplet. For example, the chiral multiplet $T\left(\bar{w}^{2}\right)$ has weights $(2,2)$. In global supersymmetry the operator $T$ corresponds to the usual chiral projection operator $\bar{D}^{2} .8$

From now on, we will drop the notation of h.c. and implicitly assume its presence for every []$_{F}$ term in the Lagrangian. Finally, the multiplet $(V)_{D}$ is a linear multiplet with weights $(2,0)$, given by

$$
(V)_{D}=\left(D, \Phi \lambda, 0, \mathscr{D}^{b} \hat{F}_{a b},-\not D \lambda,-\square^{C} D\right)
$$

The definitions of $\Phi \lambda$ and the covariant field strength $\hat{F}_{a b}$ can be found in eq. (17.1) of [23], which reduce for an abelian gauge field to

$$
\begin{aligned}
\hat{F}_{a b} & =e_{a}^{\mu} e_{b}^{v}\left(2 \partial_{[\mu} A_{v]}+\bar{\psi}_{[\mu} \gamma_{v]} \lambda\right) \\
\mathscr{D}_{\mu} \lambda & =\left(\partial_{\mu}-\frac{3}{2} b_{\mu}+\frac{1}{4} w_{\mu}^{a b} \gamma_{a b}-\frac{3}{2} i \gamma_{*} \mathscr{A}_{\mu}\right) \lambda-\left(\frac{1}{4} \gamma^{a b} \hat{F}_{a b}+\frac{1}{2} i \gamma_{*} D\right) \psi_{\mu} .
\end{aligned}
$$

Here, $e_{a}^{\mu}$ is the vierbein, with frame indices $a, b$ and coordinate indices $\mu, v$. The fields $w_{\mu}^{a b}, b_{\mu}$, and $\mathscr{A}_{\mu}$ are the gauge fields corresponding to Lorentz transformations, dilatations, and $T_{R}$ symmetry of the conformal algebra respectively, while $\psi_{\mu}$ is the gravitino. The conformal d'Alembertian is given by $\square^{C}=\eta^{a b} \mathscr{D}_{a} \mathscr{D}_{b}$.

It is important to note that the FI term given by eq. (5.1) does not require the gauging of an R-symmetry, but breaks invariance under Kähler transformations. In fact, a gauged R-symmetry would forbid such a term $\mathscr{L}_{F I}$ [19]. ${ }^{9}$

The resulting Lagrangian after integrating out the auxiliary field $D$ contains a term

$$
\mathscr{L}_{\text {FI,new }}=-\frac{\xi_{2}^{2}}{2}\left(s_{0} \bar{s}_{0}\right)^{2} .
$$

In the absence of additional matter fields, one can use the Poincaré gauge $s_{0}=\bar{s}_{0}=1$, resulting in a constant D-term contribution to the scalar potential. This prefactor however is relevant when matter couplings are included in the next section.

\footnotetext{
${ }^{8}$ The operator $T$ indeed has the property that $T(Z)=0$ for a chiral multiplet $Z$. Moreover, for a vector multiplet $V$ we have $T(Z C)=Z T(C)$, and $[C]_{D}=\frac{1}{2}[T(C)]_{F}$.

${ }^{9}$ We kept the notation of [19]. Note that in this notation the field strength superfield $\mathscr{W}_{\alpha}$ is given by $\mathscr{W}^{2}=\bar{\lambda} P_{L} \lambda$, and $(V)_{D}$ corresponds to $\mathscr{D}^{\alpha} \mathscr{W}_{\alpha}$.
} 


\subsection{Adding (charged) matter fields}

In this section we couple the term $\mathscr{L}_{\text {FI }}$ given by eq. (5.1) to additional matter fields charged under the $U(1)$. For simplicity, we focus on a single chiral multiplet $X$. The extension to more chiral multiplets is trivial. The Lagrangian is given by

$$
\mathscr{L}=-3\left[S_{0} \bar{S}_{0} e^{-\frac{1}{3} K(X, \bar{X})}\right]_{D}+\left[S_{0}^{3} W(X)\right]_{F}-\frac{1}{4}\left[f(X) \bar{\lambda} P_{L} \lambda\right]_{F}+\mathscr{L}_{\mathrm{FI}},
$$

with a Kähler potential $K(X, \bar{X})$, a superpotential $W(X)$ and a gauge kinetic function $f(X)$. The first three terms in eq. (5.8) give the usual supergravity Lagrangian [23]. We assume that the multiplet $X$ transforms under the $U(1)$,

$$
\begin{aligned}
& V \rightarrow V+\Lambda+\bar{\Lambda} \\
& X \rightarrow X e^{-q \Lambda}
\end{aligned}
$$

with gauge multiplet parameter $\Lambda$. We assume that the $U(1)$ is not an R-symmetry. In other words, we assume that the superpotential does not transform under the gauge symmetry. For a model with a single chiral multiplet this implies that the superpotential is constant

$$
W(X)=F
$$

Gauge invariance fixes the Kähler potential to be a function of $X e^{q V} \bar{X}$ (for notational simplicity, in the following we omit the $e^{q V}$ factors).

Indeed, in this case the term $\mathscr{L}_{\mathrm{FI}}$ can be consistently added to the theory, similar to [19], and the resulting D-term contribution to the scalar potential acquires an extra term proportional to $\xi_{2}$

$$
\mathscr{V}_{D}=\frac{1}{2} \operatorname{Re}(f(X))^{-1}\left(i k_{X} \partial_{X} K+\xi_{2} e^{\frac{2}{3} K}\right)^{2},
$$

where the Killing vector is $k_{X}=-i q X$ and $f(X)$ is the gauge kinetic function. The F-term contribution to the scalar potential remains the usual

$$
\mathscr{V}_{F}=e^{K(X, \bar{X})}\left(-3 W \bar{W}+g^{X \bar{X}} \nabla_{X} W \bar{\nabla}_{\bar{X}} \bar{W}\right) .
$$

For a constant superpotential (5.10) this reduces to

$$
\mathscr{V}_{F}=|F|^{2} e^{K(X, \bar{X})}\left(-3+g^{X \bar{X}} \partial_{X} K \partial_{\bar{X}} K\right) .
$$

From eq. (5.11) it can be seen that if the Kähler potential includes a term proportional to $\xi_{1} \log (X \bar{X})$, the D-term contribution to the scalar potential acquires another constant contribution. For example, if

$$
K(X, \bar{X})=X \bar{X}+\xi_{1} \ln (X \bar{X}),
$$

the D-term contribution to the scalar potential becomes

$$
\mathscr{V}_{D}=\frac{1}{2} \operatorname{Re}(f(X))^{-1}\left(q X \bar{X}+q \xi_{1}+\xi_{2} e^{\frac{2}{3} K}\right)^{2} .
$$


In fact the contribution proportional to $\xi_{1}$ is the usual FI term in a non R-symmetric Kähler frame, which can be consistently added to the model including the new FI term proportional to $\xi_{2}$.

In the absence of the extra term, a Kähler transformation

$$
\begin{aligned}
K(X, \bar{X}) & \rightarrow K(X, \bar{X})+J(X)+\bar{J}(\bar{X}), \\
W(X) & \rightarrow W(X) e^{-J(X)},
\end{aligned}
$$

with $J(X)=-\xi_{1} \ln X$ allows one to recast the model in the form

$$
\begin{aligned}
K(X, \bar{X}) & =X \bar{X}, \\
W(X) & =m_{3 / 2} X .
\end{aligned}
$$

The two models result in the same Lagrangian, at least classically ${ }^{10}$. However, in the Kähler frame of eqs. (5.17) the superpotential transforms nontrivially under the gauge symmetry. As a consequence, the gauge symmetry becomes an R-symmetry. Note that [18]:

1. The extra term (5.1) violates the Kähler invariance of the theory, and the two models related by a Kähler transformation are no longer equivalent.

2. The model written in the Kähler frame where the gauge symmetry becomes an R-symmetry in eqs. (5.17) can not be consistently coupled to $\mathscr{L}_{\text {FI }}$.

\section{The scalar potential in a Non R-symmetry frame}

In this section, we work in the Kähler frame where the superpotential does not transform, and take into account the two types of FI terms which were discussed in the last section. For convenience, we repeat here the Kähler potential in eq. (5.14) and restore the inverse reduced Planck mass $\kappa=M_{\mathrm{Pl}}^{-1}=\left(2.4 \times 10^{18} \mathrm{GeV}\right)^{-1}$ :

$$
K=\kappa^{-2}\left(X \bar{X}+\xi_{1} \ln X \bar{X}\right) .
$$

The superpotential and the gauge kinetic function are set to be constant ${ }^{11}$ :

$$
W=\kappa^{-3} F, \quad f(X)=1 .
$$

After performing a change of the field variable $X=\rho e^{i \theta}$ where $\rho \geq 0$ and setting $\xi_{1}=b$, the full scalar potential $\mathscr{V}=\mathscr{V}_{F}+\mathscr{V}_{D}$ is a function of $\rho$. The F-term contribution to the scalar potential is given by

$$
\mathscr{V}_{F}=\frac{1}{\kappa^{4}} F^{2} e^{\rho^{2}} \rho^{2 b}\left[\frac{\left(b+\rho^{2}\right)^{2}}{\rho^{2}}-3\right],
$$

\footnotetext{
${ }^{10}$ At the quantum level, a Kähler transformation also introduces a change in the gauge kinetic function $f$, see for example [36].

${ }^{11}$ Strictly speaking, the gauge kinetic function gets a field-dependent correction proportional to $q^{2} \ln \rho$, in order to cancel the chiral anomalies [11]. However, the correction turns out to be very small and can be neglected below, since the charge $q$ is chosen to be of order of $10^{-5}$ or smaller.
} 
and the D-term contribution is

$$
\mathscr{V}_{D}=\frac{q^{2}}{2 \kappa^{4}}\left(b+\rho^{2}+\xi \rho^{\frac{4 b}{3}} e^{\frac{2}{3} \rho^{2}}\right)^{2} .
$$

Note that we rescaled the second FI parameter by $\xi=\xi_{2} / q$. We consider the case with $\xi \neq 0$ because we are interested in the role of the new FI-term in inflationary models driven by supersymmetry breaking. Moreover, the limit $\xi \rightarrow 0$ is ill-defined [19].

The first FI parameter $b$ was introduced as a free parameter. We now proceed to narrowing the value of $b$ by the following physical requirements. We first consider the behaviour of the potential around $\rho=0$,

$$
\begin{aligned}
& \mathscr{V}_{D}=\frac{q^{2}}{2 \kappa^{4}}\left[\left(b^{2}+2 b \rho^{2}+O\left(\rho^{4}\right)\right)+2 b \xi \rho^{\frac{4 b}{3}}\left(1+O\left(\rho^{2}\right)\right)+\xi^{2} \rho^{\frac{8 b}{3}}\left(1+O\left(\rho^{2}\right)\right)\right], \\
& \mathscr{V}_{F}=\frac{F^{2}}{\kappa^{4}} \rho^{2 b}\left[b^{2} \rho^{-2}+(2 b-3)+O\left(\rho^{2}\right)\right] .
\end{aligned}
$$

Here we are interested in small-field inflation models in which the inflation starts in the neighbourhood of a local maximum at $\rho=0$. In [16], we considered models of this type with $\xi=0$ (which were called Case 1 models), and found that the choice $b=1$ is forced by the requirement that the potential takes a finite value at the local maximum $\rho=0$. Now, we will investigate the effect of the new FI parameter $\xi$ on the choice of $b$ under the same requirement.

First, in order for $\mathscr{V}(0)$ to be finite, we need $b \geq 0$. We first consider the case $b>0$. We next investigate the condition that the potential at $\rho=0$ has a local maximum. For clarity we discuss below the cases of $F=0$ and $F \neq 0$ separately. The $b=0$ case will be treated at the end of this section.

\subsection{Case $F=0$}

In this case $\mathscr{V}_{F}=0$ and the scalar potential is given by only the D-term contribution $\mathscr{V}=\mathscr{V}_{D}$. Let us first discuss the first derivative of the potential:

$$
\mathscr{V}_{D}^{\prime}=\frac{q^{2}}{2 \kappa^{4}}\left[4 b \rho\left(1+O\left(\rho^{2}\right)\right)+\frac{8 b^{2}}{3} \xi \rho^{\frac{4 b}{3}-1}\left(1+O\left(\rho^{2}\right)\right)+\frac{8 b}{3} \xi^{2} \rho^{\frac{8 b}{3}-1}\left(1+O\left(\rho^{2}\right)\right)\right] .
$$

For $\mathscr{V}_{D}^{\prime}(0)$ to be convergent, we need $b \geq 3 / 4$ (note that $\xi \neq 0$ ). When $b=3 / 4$, we have $\mathscr{V}_{D}^{\prime}(0)=$ $8 b^{2} \xi / 3$, which does not give an extremum because we chose $\xi \neq 0$. On the other hand, when $b>3 / 4$, we have $\mathscr{V}_{D}^{\prime}(0)=0$. To narrow the allowed value of $b$ further, let us turn to the second derivative,

$$
\begin{aligned}
\mathscr{V}_{D}^{\prime \prime}=\frac{q^{2}}{2 \kappa^{4}}[4 b(1 & \left.+O\left(\rho^{2}\right)\right)+\frac{8 b^{2}}{3}\left(\frac{4 b}{3}-1\right) \xi \rho^{\frac{4 b}{3}-2}\left(1+O\left(\rho^{2}\right)\right) \\
& \left.+\frac{8 b}{3}\left(\frac{8 b}{3}-1\right) \xi^{2} \rho^{\frac{8 b}{3}-2}\left(1+O\left(\rho^{2}\right)\right)\right] .
\end{aligned}
$$

When $3 / 4<b<3 / 2$, the second derivative $\mathscr{V}_{D}^{\prime \prime}(0)$ diverges. When $b>3 / 2$, the second derivative becomes $\mathscr{V}_{D}^{\prime \prime}(0)=2 \kappa^{-4} q^{2} b>0$, which gives a minimum. 
We therefore conclude that to have a local maximum at $\rho=0$, we need to choose $b=3 / 2$, for which we have

$$
\mathscr{V}_{D}^{\prime \prime}(0)=3 \kappa^{-4} q^{2}(\xi+1)
$$

The condition that $\rho=0$ is a local maximum requires $\xi<-1$.

Let us next discuss the global minimum of the potential with $b=3 / 2$ and $\xi<-1$. The first derivative of the potential without approximation reads

$$
\mathscr{V}_{D}^{\prime} \propto \rho\left(3+3 \xi e^{\frac{2}{3} \rho^{2}}+2 \xi \rho^{2} e^{\frac{2}{3} \rho^{2}}\right)\left(3+2 \rho^{2}+2 \xi \rho^{2} e^{\frac{2}{3} \rho^{2}}\right) .
$$

Since $3+3 \xi e^{\frac{2}{3} \rho^{2}}+2 \xi \rho^{2} e^{\frac{2}{3} \rho^{2}}<0$ for $\rho \geq 0$ and $\xi<-1$, the extremum away from $\rho=0$ is located at $\rho_{v}$ satisfying the condition

$$
3+2 \rho_{v}^{2}+2 \xi \rho_{v}^{2} e^{\frac{2}{3} \rho_{v}^{2}}=0
$$

Substituting this condition into the potential $\mathscr{V}_{D}$ gives $\mathscr{V}_{D}\left(\rho_{v}\right)=0$.

We conclude that for $\xi<-1$ and $b=3 / 2$ the potential has a maximum at $\rho=0$, and a supersymmetric minimum at $\rho_{v}$. We postpone the analysis of inflation near the maximum of the potential in section 7 , and the discussion of the uplifting of the minimum in order to obtain a small but positive cosmological constant below. In the next subsection we investigate the case $F \neq 0$.

We finally comment on supersymmetry (SUSY) breaking in the scalar potential. Since the superpotential is zero, the SUSY breaking is measured by the D-term order parameter, namely the Killing potential associated with the gauged $\mathrm{U}(1)$, which is defined by

$$
\mathscr{D}=i \kappa^{-2} \frac{-i q X}{W}\left(\frac{\partial W}{\partial X}+\kappa^{2} \frac{\partial \mathscr{K}}{\partial X} W\right)
$$

This enters the scalar potential as $\mathscr{V}_{D}=\mathscr{D}^{2} / 2$. So, at the local maximum and during inflation $\mathscr{D}$ is of order $q$ and supersymmetry is broken. On the other hand, at the global minimum, supersymmetry is preserved and the potential vanishes.

\subsection{Case $F \neq 0$}

In this section we take into account the effect of $\mathscr{V}_{F}$; its first derivative reads:

$$
\mathscr{V}_{F}^{\prime}=\kappa^{-4} F^{2}\left[b^{2}(2 b-2) \rho^{2 b-3}+2 b(2 b-3) \rho^{2 b-1}\left(1+O\left(\rho^{2}\right)\right)\right] .
$$

For $\mathscr{V}^{\prime}(0)$ to be convergent, we need $b \geq 3 / 2$, for which $\mathscr{V}_{D}^{\prime}(0)=0$ holds. For $b=3 / 2$, we have $\mathscr{V}_{F}^{\prime}(0)=(9 / 4) \kappa^{-4} F^{2}>0$, that does not give an extremum. For $b>3 / 2$, we have $\mathscr{V}_{F}^{\prime}(0)=0$. To narrow the allowed values of $b$ further, let us turn to the second derivative,

$$
\mathscr{V}_{F}^{\prime \prime}=\kappa^{-4} F^{2}\left[b^{2}(2 b-2)(2 b-3) \rho^{2 b-4}+2 b(2 b-3)(2 b-1) \rho^{2 b-2}\left(1+O\left(\rho^{2}\right)\right)\right] .
$$

For $3 / 2<b<2$, the second derivative $\mathscr{V}_{F}^{\prime \prime}(0)$ diverges. For $b \geq 2$, the second derivative is positive $\mathscr{V}^{\prime \prime}(0)>0$, that gives a minimum (note that $V_{D}^{\prime \prime}(0)>0$ as well in this range). 
We conclude that the potential cannot have a local maximum at $\rho=0$ for any choice of $b$. Nevertheless, as we will show below, the potential can have a local maximum in the neighbourhood of $\rho=0$ if we choose $b=3 / 2$ and $\xi<-1$. For this choice, the derivatives of the potential have the following properties,

$$
\mathscr{V}^{\prime}(0)<0, \quad \mathscr{V}^{\prime \prime}(0)=3 \kappa^{-4} q^{2}(\xi+1)
$$

The extremisation condition around $\rho=0$ becomes

$$
3 \kappa^{-4} q^{2}(\xi+1) \rho+\frac{9}{4} \kappa^{-4} F^{2} \simeq 0 .
$$

So the extremum is at

$$
\rho \simeq-\frac{3 F^{2}}{4 q^{2}(\xi+1)}
$$

Note that the extremum is in the neighbourhood of $\rho=0$ as long as we keep the $F$-contribution to the scalar potential small by taking $F^{2} \ll q^{2}|\xi+1|$, which guarantees the approximation ignoring higher order terms in $\rho$. We now choose $\xi<-1$ so that $\rho$ for this extremum is positive. The second derivative at the extremum reads

$$
\mathscr{V}^{\prime \prime} \simeq 3 \kappa^{-4} q^{2}(\xi+1),
$$

as long as we ignore higher order terms in $F^{2} /\left(q^{2}|\xi+1|\right)$. By our choice $\xi<-1$, the extremum is a local maximum, as desired.

Let us comment on the global minimum after turning on the F-term contribution. As long as we choose the parameters so that $F^{2} / q^{2} \ll 1$, the change in the global minimum $\rho_{v}$ is very small, of order $\mathscr{O}\left(F^{2} / q^{2}\right)$, because the extremisation condition depends only on the ratio $F^{2} / q^{2}$. So the change in the value of the global minimum is of order $\mathscr{O}\left(F^{2}\right)$. The plot of this change is given in Fig. 2.

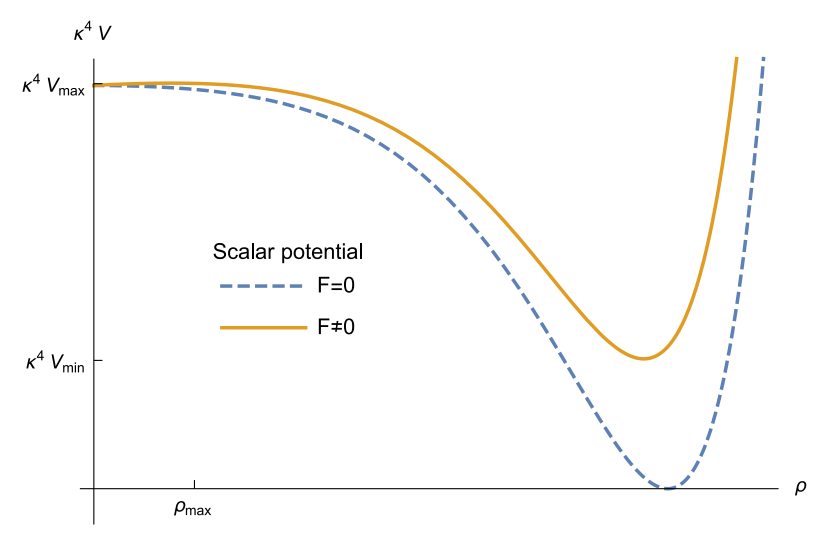

Figure 2: This plot shows the scalar potentials in $F=0$ and $F \neq 0$ cases. When $F=0$, we have a local maximum at $\rho_{\max }=0$ and a global minimum with zero cosmological constant. For $F \neq 0$, the local maximum is shifted by a small positive value to $\rho_{\max } \neq 0$. The global minimum now has a positive cosmological constant. 
In the present case $F \neq 0$, the order parameters of SUSY breaking are both the Killing potential $\mathscr{D}$ and the F-term contribution $\mathscr{F}_{X}$, which read

$$
\mathscr{D} \propto q\left(\frac{3}{2}+\rho^{2}\right), \quad \mathscr{F}_{X} \propto F \rho^{1 / 2} e^{\rho^{2} / 2},
$$

where the F-term order parameter $\mathscr{F}_{X}$ is defined by

$$
\mathscr{F}_{X}=-\frac{1}{\sqrt{2}} e^{\kappa^{2} \mathscr{K} / 2}\left(\frac{\partial^{2} \mathscr{K}}{\partial X \partial \bar{X}}\right)^{-1}\left(\frac{\partial \bar{W}}{\partial \bar{X}}+\kappa^{2} \frac{\partial \mathscr{K}}{\partial \bar{X}} \bar{W}\right) .
$$

Therefore, at the local maximum, $\mathscr{F}_{X} / \mathscr{D}$ is of order $\mathscr{O}\left((\xi+1)^{-1 / 2} F^{2} / q^{2}\right)$ because $\rho$ there is of order $\mathscr{O}\left((\xi+1)^{-1} F^{2} / q^{2}\right)$. On the other hand, at the global minimum, both $\mathscr{D}$ and $\mathscr{F}_{X}$ are of order $\mathscr{O}(F)$, assuming that $\rho$ at the minimum is of order $\mathscr{O}(1)$, which is true in our models below. This makes tuning of the vacuum energy between the F- and D-contribution in principle possible, along the lines of $[16,11]$.

A comment must be made here on the action in the presence of non-vanishing $F$ and $\xi$. As mentioned above, the supersymmetry is broken both by the gauge sector and by the matter sector. The associated goldstino therefore consists of a linear combination of the $U(1)$ gaugino and the fermion in the matter chiral multiplet $X$. In the unitary gauge the goldstino is set to zero, so the gaugino is not vanishing anymore, and the action does not simplify as in Ref. [19]. This, however, only affects the part of the action with fermions, while the scalar potential does not change. This is why we nevertheless used the scalar potential (6.3) and (6.4).

Let us consider now the case $b=0$ where only the new FI parameter $\xi$ contributes to the potential. In this case, the condition for the local maximum of the scalar potential at $\rho=0$ can be satisfied for $-\frac{3}{2}<\xi<0$. When $F$ is set to zero, the scalar potential (6.4) has a minimum at $\rho_{\min }^{2}=\frac{3}{2} \ln \left(-\frac{3}{2 \xi}\right)$. In order to have $\mathscr{V}_{\min }=0$, we can choose $\xi=-\frac{3}{2 e}$. However, we find that this choice of parameter $\xi$ does not allow slow-roll inflation near the maximum of the scalar potential. Similar to the previous model of section 4, it may be possible to achieve both the scalar potential satisfying slow-roll conditions and a small cosmological constant at the minimum by adding correction terms to the Kähler potential and turning on a parameter $F$. However, here, we will focus on $b=3 / 2$ case where, as we will see shortly, less parameters are required to satisfy the observational constraints.

\section{Application in Inflation}

We recall that the the models we described in section 4, the inflaton is identified with the sgoldstino, carrying a $U(1)$ charge under a gauged R-symmetry and inflation occurs around the maximum of the scalar potential, where the $U(1)$ symmetry is restored, with the inflaton rolling down towards the electroweak minimum. These models avoid the so-called $\eta$-problem in supergravity by taking a linear superpotential, $W \propto X$. In contrast, here we will consider models with two FI parameters $b, \xi$ in the Kähler frame where the $U(1)$ gauge symmetry is not an R-symmetry. If the new FI term $\xi$ is zero, these models are Kähler equivalent to those with a linear superpotential (Case 1 models with $b=1$ ). The presence of non-vanishing $\xi$, however, breaks the Kähler invariance as we discussed before. Moreover, the FI parameter $b$ cannot be 1 but is forced to be 
$b=3 / 2$, according to the argument in Section 6. So the new models do not seem to avoid the $\eta$-problem. Nevertheless, we will show below that this is not the case and the new models with $b=3 / 2$ avoid the $\eta$-problem thanks to the other FI parameter $\xi$ which is chosen near the value at which the effective charge of $X$ vanishes between the two FI-terms. Inflation is again driven from supersymmetry breaking but from a D-term rather than an F-term as we had before.

\subsection{Example for slow-roll D-term inflation}

In this section we focus on the case where $b=3 / 2$ and derive the condition that leads to slowroll inflation scenarios, where the start of inflation (or, horizon crossing) is near the maximum of the potential at $\rho=0$. We also assume that the scalar potential is D-term dominated by choosing $F=0$, for which the model has only two parameters, namely $q$ and $\xi$. The parameter $q$ controls the overall scale of the potential and it will be fixed by the amplitude $A_{s}$ of the CMB data. The only free-parameter left over is $\xi$, which can be tuned to satisfy the slow-roll condition.

In order to calculate the slow-roll parameters, we need to work with the canonically normalised field $\chi$ defined by eqs. (4.11), (4.12). Since we assume inflation to start near $\rho=0$, the slow-roll parameters for small $\rho$ can be expanded as

$$
\begin{aligned}
\varepsilon & =\frac{F^{4}}{q^{4}}+\frac{4 F^{2}\left(2(\xi+1) q^{4}-3 F^{4}\right)}{3 q^{6}} \rho \\
& +\left(\frac{16}{9}(\xi+1)^{2}+\frac{2 F^{4}\left(18 F^{4}-q^{4}(20 \xi+11)\right)}{3 q^{8}}\right) \rho^{2}+\mathscr{O}\left(\rho^{3}\right), \\
\eta & =\frac{4(1+\xi)}{3}+\mathscr{O}(\rho) .
\end{aligned}
$$

Note also that $\eta$ is negative when $\xi<-1$. We can therefore tune the parameter $\xi$ to avoid the $\eta$-problem. The observation is that at $\xi=-1$, the effective charge of $X$ vanishes and thus the $\rho$-dependence in the $\mathrm{D}$-term contribution (6.4) becomes of quartic order.

For our present choice $F=0$, the potential and the slow-roll parameters become functions of $\rho^{2}$ and the slow-roll parameters for small $\rho^{2}$ read

$$
\begin{aligned}
& \eta=\frac{4(1+\xi)}{3}+\mathscr{O}\left(\rho^{2}\right), \\
& \varepsilon=\frac{16}{9}(\xi+1)^{2} \rho^{2}+\mathscr{O}\left(\rho^{4}\right) \simeq \eta(0)^{2} \rho^{2}
\end{aligned}
$$

Note that we obtain the same relation between $\varepsilon$ and $\eta$ as in the model of inflation from supersymmetry breaking driven by an F-term from a linear superpotential and $b=1$ (see eq. (4.13)). Thus, there is a possibility to have flat plateau near the maximum that satisfies the slow-roll condition and at the same time a small cosmological constant at the minimum nearby.

The number of e-folds $N$ during inflation is determined by

$$
N=\kappa^{2} \int_{\chi_{*}}^{\chi_{\text {end }}} \frac{\mathscr{V}}{\partial_{\chi} \mathscr{V}} d \chi=\kappa^{2} \int_{\rho_{*}}^{\rho_{\text {end }}} \frac{\mathscr{V}}{\partial_{\rho} \mathscr{V}}\left(\frac{d \chi}{d \rho}\right)^{2} d \rho
$$

where we choose $\left|\varepsilon\left(\chi_{\text {end }}\right)\right|=1$. Notice that the slow-roll parameters for small $\rho^{2}$ satisfy the simple relation $\varepsilon=\eta(0)^{2} \rho^{2}+O\left(\rho^{4}\right)$ by eq. (7.2). Therefore, the number of e-folds between $\rho=\rho_{1}$ and 
$\rho_{2}\left(\rho_{1}<\rho_{2}\right)$ takes the following simple approximate form as in (4.19):

$$
N \simeq \frac{1}{|\eta(0)|} \ln \left(\frac{\rho_{2}}{\rho_{1}}\right)=\frac{3}{4|\xi+1|} \ln \left(\frac{\rho_{2}}{\rho_{1}}\right) .
$$

as long as the expansions in (7.2) are valid in the region $\rho_{1} \leq \rho \leq \rho_{2}$. Here we also used the approximation $\eta(0) \simeq \eta_{*}$, which holds in this approximation.

We can compare the theoretical predictions of our model to the observational data via the power spectrum of scalar perturbations of the CMB, namely the amplitude $A_{s}$, tilt $n_{s}$ and the tensorto-scalar ratio of primordial fluctuations $r$. These are written in terms of the slow-roll parameters:

$$
\begin{aligned}
A_{s} & =\frac{\kappa^{4} \mathscr{V}_{*}}{24 \pi^{2} \varepsilon_{*}}, \\
n_{s} & =1+2 \eta_{*}-6 \varepsilon_{*} \simeq 1+2 \eta_{*}, \\
r & =16 \varepsilon_{*},
\end{aligned}
$$

where all parameters are evaluated at the field value at horizon crossing $\chi_{*}$. From the relation of the spectral index above, one should have $\eta_{*} \simeq-0.02$, and thus eq. (7.4) gives approximately the desired number of e-folds when the logarithm is of order one. Actually, using this formula, we can estimate the upper bound of the tensor-to-scalar ratio $r$ and the Hubble scale $H_{*}$ following the same argument given in section 4; that is, the upper bounds are given by computing the parameters $r, H_{*}$ assuming that the expansions (7.2) hold until the end of inflation. We then get the bound

$$
r \lesssim 16\left(\left|\eta_{*}\right| \rho_{\mathrm{end}} e^{-\left|\eta_{*}\right| N}\right)^{2} \simeq 10^{-4}, \quad H_{*} \lesssim 10^{12} \mathrm{GeV}
$$

where we used $\left|\eta_{*}\right|=0.02, N \simeq 50-60$ and $\rho_{\text {end }} \lesssim 0.5$, which are consistent with our models. In the next subsection, we will present a model which gives a tensor-to-scalar ratio bigger than the upper bound above, by adding some perturbative corrections to the Kähler potential.

As an example, let us consider the case where

$$
q=4.544 \times 10^{-7}, \quad \xi=-1.005 .
$$

By choosing the initial condition $\rho_{*}=0.055$ and $\rho_{\text {end }}=0.403$, we obtain the results $N=58$, $n_{s}=0.9542, r=7.06 \times 10^{-6}$ and $A_{s}=2.2 \times 10^{-9}$, which are within the $2 \sigma$-region of Planck' 15 data [18].

As was shown in Section 6.1, this model has a supersymmetric minimum with zero cosmological constant because $F$ is chosen to be zero. One possible way to generate a non-zero cosmological constant at the minimum is to turn on the superpotential $W=\kappa^{-3} F \neq 0$, as mentioned in Section 6.2. In this case, the scale of the cosmological constant is of order $\mathscr{O}\left(F^{2}\right)$. It would be interesting to find an inflationary model which has a minimum at a tiny tuneable vacuum energy with a supersymmetry breaking scale consistent with the low energy particle physics.

\subsection{A small field inflation model from supergravity with observable tensor-to-scalar ratio}

While the results in the previous example agree with the current limits on $r$ set by Planck, supergravity models with higher $r$ are of particular interest. In this section we show that our model 
can get large $r$ at the price of introducing some additional terms in the Kähler potential. Let us consider the previous model with additional quadratic and cubic terms in $X \bar{X}$ :

$$
K=\kappa^{-2}\left(X \bar{X}+A(X \bar{X})^{2}+B(X \bar{X})^{3}+b \ln X \bar{X}\right),
$$

while the superpotential and the gauge kinetic function remain as in eq. (6.2). We now assume that inflation is driven by the D-term, setting the parameter $F=0$. In terms of the field variable $\rho$, we obtain the scalar potential:

$$
\mathscr{V}=q^{2}\left(b+\rho^{2}+2 A \rho^{4}+3 B \rho^{6}+\xi \rho^{\frac{4 b}{3}} e^{\frac{2}{3}\left(A \rho^{4}+B \rho^{6}+\rho^{2}\right)}\right)^{2} .
$$

We thus have two more parameters $A$ and $B$. This does not affect the arguments of the choices of $b$ in the previous sections because these parameters appear in higher orders in $\rho$ in the scalar potential. So, we consider the case $b=3 / 2$. The simple formula (7.4) for the number of e-folds for small $\rho^{2}$ also holds even when $A, B$ are turned on because the new parameters appear at order $\rho^{4}$ and higher. To obtain $r \approx 0.01$, we can choose for example

$$
q=2.121 \times 10^{-5}, \quad \xi=-1.140, \quad A=0.545, \quad B=0.230 .
$$

By choosing the initial condition $\rho_{*}=0.240$ and $\rho_{\text {end }}=0.720$, we obtain the results $N=57$, $n_{s}=0.9603, r=0.015$ and $A_{s}=2.2 \times 10^{-9}$, which agree with Planck'15 data as shown in Fig. 3 .

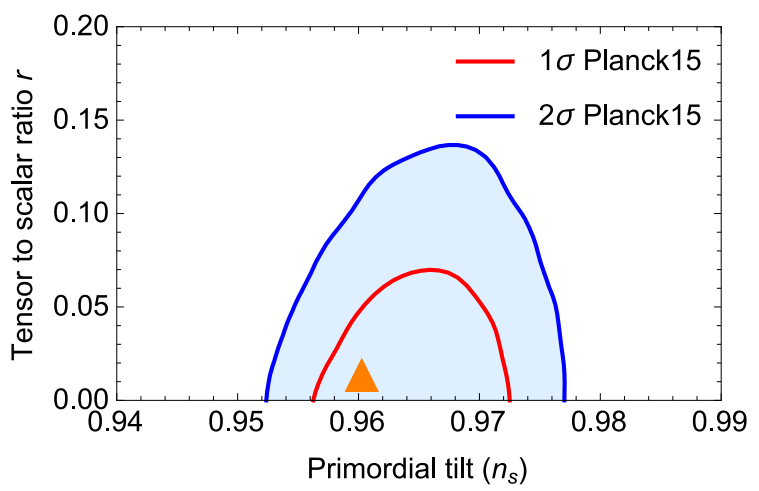

Figure 3: A plot of the predictions for the scalar potential with $F=0, b=3 / 2, A=0.545, B=0.230$, $\xi=-1.140$ and $q=2.121 \times 10^{-5}$ in the $n_{s}-r$ plane, versus Planck' 15 results.

In summary, in contrast to the model in section 4, where the F-term contribution is dominant during inflation, here inflation is driven purely by a D-term. Moreover, a canonical Kähler potential (6.1) together with two FI-parameters ( $q$ and $\xi$ ) is enough to satisfy Planck' 15 constraints, and no higher order correction to the Kähler potential is needed. However, to obtain a larger tensor-toscalar ratio, we have to introduce perturbative corrections to the Kähler potential up to cubic order in $X \bar{X}$ (i.e. up to order $\rho^{6}$ ). This model provides a supersymmetric extension of the model [37], which realises large $r$ at small field inflation without referring to supersymmetry.

\section{Acknowledgements}

This work was supported in part by the Swiss National Science Foundation and in part by a CNRS PICS grant. 


\section{References}

[1] I. Antoniadis, N. Arkani-Hamed, S. Dimopoulos and G. R. Dvali, "New dimensions at a millimeter to a Fermi and superstrings at a TeV,” Phys. Lett. B 436 (1998) 257 [arXiv:hep-ph/9804398].

[2] I. Antoniadis and S. P. Patil, Eur. Phys. J. C75 (2015) 182 [arXiv:1410.8845 [hep-th]].

[3] I. Antoniadis and R. Knoops, Nucl. Phys. B 886 (2014) 43 [arXiv:1403.1534 [hep-th]]; G. Villadoro and F. Zwirner, Phys. Rev. Lett. 95 (2005) 231602 [hep-th/0508167].

[4] P. Fayet and J. Iliopoulos, "Spontaneously Broken Supergauge Symmetries and Goldstone Spinors," Phys. Lett. 51B (1974) 461; P. Fayet, Phys. Lett. B 69 (1977) 489.

[5] I. Antoniadis, D. M. Ghilencea and R. Knoops, JHEP 1502 (2015) 166 [arXiv:1412.4807 [hep-th]].

[6] I. Antoniadis and R. Knoops, Nucl. Phys. B 902 (2016) 69 [arXiv:1507.06924 [hep-ph]].

[7] L. Randall and R. Sundrum, Nucl. Phys. B 557 (1999) 79 [hep-th/9810155]; G. F. Giudice, M. A. Luty, H. Murayama and R. Rattazzi, JHEP 9812 (1998) 027 [hep-ph/9810442].

[8] J. A. Bagger, T. Moroi and E. Poppitz, JHEP 0004 (2000) 009 [hep-th/9911029].

[9] I. Antoniadis and T. Maillard, "Moduli stabilization from magnetic fluxes in type I string theory," Nucl. Phys. B 716 (2005) 3 [hep-th/0412008]; I. Antoniadis, A. Kumar and T. Maillard, "Magnetic fluxes and moduli stabilization," Nucl. Phys. B 767 (2007) 139 [hep-th/0610246].

[10] I. Antoniadis, J.-P. Derendinger and T. Maillard, "Nonlinear N=2 Supersymmetry, Effective Actions and Moduli Stabilization,” Nucl. Phys. B 808 (2009) 53 [arXiv:0804.1738 [hep-th]].

[11] I. Antoniadis, A. Chatrabhuti, H. Isono and R. Knoops, "Inflation from Supergravity with Gauged R-symmetry in de Sitter Vacuum,” Eur. Phys. J. C 76 (2016) no.12, 680 [arXiv:1608.02121 [hep-ph]].

[12] A. H. Guth, "The Inflationary Universe: A Possible Solution to the Horizon and Flatness Problems," Phys. Rev. D 23 (1981) 347; A. D. Linde, "A New Inflationary Universe Scenario: A Possible Solution of the Horizon, Flatness, Homogeneity, Isotropy and Primordial Monopole Problems," Phys. Lett. 108B (1982) 389; A. Albrecht and P. J. Steinhardt, "Cosmology for Grand Unified Theories with Radiatively Induced Symmetry Breaking,” Phys. Rev. Lett. 48 (1982) 1220.

[13] D. H. Lyth and A. Riotto, "Particle physics models of inflation and the cosmological density perturbation," Phys. Rept. 314 (1999) 1 [hep-ph/9807278]; A. D. Linde, "Particle physics and inflationary cosmology,” Contemp. Concepts Phys. 5 (1990) 1 [hep-th/0503203].

[14] A. A. Starobinsky, “A New Type of Isotropic Cosmological Models Without Singularity,” Phys. Lett. 91B (1980) 99.

[15] L. Randall and S. D. Thomas, "Solving the cosmological moduli problem with weak scale inflation," Nucl. Phys. B 449 (1995) 229 [hep-ph/9407248]; A. Riotto, "Inflation and the nature of supersymmetry breaking,” Nucl. Phys. B 515 (1998) 413 [hep-ph/9707330]; K. I. Izawa, “Supersymmetry - breaking models of inflation,” Prog. Theor. Phys. 99 (1998) 157 [hep-ph/9708315]; W. Buchmuller, L. Covi and D. Delepine, “Inflation and supersymmetry breaking," Phys. Lett. B 491 (2000) 183 [hep-ph/0006168].

[16] I. Antoniadis, A. Chatrabhuti, H. Isono and R. Knoops, "Inflation from Supersymmetry Breaking," Eur. Phys. J. C 77 (2017) no.11, 724 [arXiv:1706.04133 [hep-th]].

[17] F. Catino, G. Villadoro and F. Zwirner, JHEP 1201 (2012) 002 [arXiv:1110.2174 [hep-th]]. 
[18] I. Antoniadis, A. Chatrabhuti, H. Isono and R. Knoops, "Fayet-Iliopoulos terms in supergravity and D-term inflation,” Eur. Phys. J. C 78 (2018) no.5, 366 [arXiv:1803.03817 [hep-th]].

[19] N. Cribiori, F. Farakos, M. Tournoy and A. Van Proeyen, "Fayet-Iliopoulos terms in supergravity without gauged R-symmetry," arXiv:1712.08601 [hep-th].

[20] Y. Aldabergenov and S. V. Ketov, "Removing instability of inflation in Polonyi-Starobinsky supergravity by adding FI term,” Mod. Phys. Lett. A 91 (2018) no.05, 1850032 [arXiv:1711.06789 [hep-th]].

[21] D. Z. Freedman and A. Van Proeyen, Cambridge, UK: Cambridge Univ. Pr. (2012) 607 p.

[22] K. Schmitz and T. T. Yanagida, "Dynamical supersymmetry breaking and late-time R symmetry breaking as the origin of cosmic inflation,” Phys. Rev. D 94 (2016) no.7, 074021 [arXiv:1604.04911 [hep-ph]].

[23] D. Z. Freedman and A. Van Proeyen, "Supergravity," Cambridge, UK: Cambridge Univ. Press (2012).

[24] P. Binetruy and G. R. Dvali, "D term inflation,” Phys. Lett. B 388 (1996) 241 [hep-ph/9606342].

[25] C. Wieck and M. W. Winkler, "Inflation with Fayet-Iliopoulos Terms," Phys. Rev. D 90 (2014) no.10, 103507 [arXiv:1408.2826 [hep-th]]; V. Domcke and K. Schmitz,“Unified model of D-term inflation," Phys. Rev. D 95 (2017) no.7, 075020 [arXiv:1702.02173 [hep-ph]].

[26] D. V. Volkov and V. P. Akulov, Is the neutrino a Goldstone particle?; Phys. Lett. B 46 (1973) 109; M. Roček, Linearizing the Volkov-Akulov model, Phys. Rev. Lett. 41 (1978) 451; U. Lindström, M. Roček, Constrained local superfields, Phys. Rev. D 19 (1979) 2300; R. Casalbuoni, S. De Curtis, D. Dominici, F. Feruglio and R. Gatto, Nonlinear realization of supersymmetry algebra from supersymmetric constraint, Phys. Lett. B 220 (1989) 569; Z. Komargodski and N. Seiberg, From linear SUSY to constrained superfields, JHEP 0909 (2009) 066; [arXiv:0907.2441 [hep-th]]; S. M. Kuzenko and S. J. Tyler, On the Goldstino actions and their symmetries, JHEP 1105, (2011) 055: [arXiv:1102.3043 [hep-th]].

[27] L. Alvarez-Gaume, C. Gomez and R. Jimenez, “Minimal Inflation,” Phys. Lett. B 690 (2010) 68 [arXiv:1001.0010 [hep-th]]; L. Alvarez-Gaume, C. Gomez and R. Jimenez, "A Minimal Inflation Scenario," JCAP 1103 (2011) 027 [arXiv:1101.4948 [hep-th]]; S. Ferrara and D. Roest, "General sGoldstino Inflation,” JCAP 1610 (2016) no.10, 038 [arXiv:1608.03709 [hep-th]].

[28] D. Baumann and D. Green, “Signatures of Supersymmetry from the Early Universe," Phys. Rev. D 85 (2012) 103520 [arXiv:1109.0292 [hep-th]].

[29] E. J. Copeland, A. R. Liddle, D. H. Lyth, E. D. Stewart and D. Wands, "False vacuum inflation with Einstein gravity," Phys. Rev. D 49 (1994) 6410 [astro-ph/9401011].

[30] D. Baumann and L. McAllister, "Inflation and String Theory," arXiv:1404.2601 [hep-th]; M. Cicoli and F. Quevedo, “String moduli inflation: An overview," Class. Quant. Grav. 28 (2011) 204001 [arXiv:1108.2659 [hep-th]].

[31] G. R. Dvali, Q. Shafi and R. K. Schaefer, "Large scale structure and supersymmetric inflation without fine tuning," Phys. Rev. Lett. 73 (1994) 1886 [hep-ph/9406319].

[32] L. Boubekeur and D. H. Lyth, “Hilltop inflation,” JCAP 0507 (2005) 010 [hep-ph/0502047].

[33] S. M. Kuzenko, "Taking a vector supermultiplet apart: Alternative Fayet-Iliopoulos-type terms," arXiv:1801.04794 [hep-th].

[34] T. Kugo and S. Uehara, " $N=1$ Superconformal Tensor Calculus: Multiplets With External Lorentz Indices and Spinor Derivative Operators,” Prog. Theor. Phys. 73 (1985) 235. 
[35] S. Ferrara, R. Kallosh, A. Van Proeyen and T. Wrase, "Linear Versus Non-linear Supersymmetry, in General,” JHEP 1604 (2016) 065 [arXiv:1603.02653 [hep-th]].

[36] V. Kaplunovsky and J. Louis, "Field dependent gauge couplings in locally supersymmetric effective quantum field theories," Nucl. Phys. B 422 (1994) 57 [hep-th/9402005].

[37] I. Wolfson and R. Brustein, "Most probable small field inflationary potentials," arXiv:1801.07057 [astro-ph]. 